\title{
Craneofaringioma en paciente pediátrico
} Reporte de un caso

Nares-López Felipe Eduardo*, Nares-López Rafael**, Reyes-González Octavio Danie***

\begin{tabular}{|c|c|}
\hline Resumen & Abstract \\
\hline $\begin{array}{l}\text { El craneofaringioma es un tumor derivado de los remanentes } \\
\text { de la bolsa de Rathke, hormonalmente inactivo, y de predomi- } \\
\text { nio supraselar hasta en el } 20 \% \text { de los casos. Representa del } \\
5 \text { al } 10 \% \text { de los tumores cerebrales de la infancia, teniendo } \\
\text { como principales síntomas la cefalea y diversas alteraciones } \\
\text { visuales y endocrinológicas. Su tratamiento es quirúrgico y es } \\
\text { recomendable la radioterapia para evitar recidivas. Se presen- } \\
\text { ta el caso de un paciente masculino de } 7 \text { años, sin anteceden- } \\
\text { tes personales patológicos de interés, que acude a consulta } \\
\text { de neurocirugía referido por endocrinología pediátrica tras es- } \\
\text { tudio por talla y peso bajo para la edad. Se realizan estudios } \\
\text { de imagen donde se confirma el diagnóstico de craneofarin- } \\
\text { gioma, el cual es tratado quirúrgicamente vía transesfenoidal, } \\
\text { resecado completamente, con lo que se mejora la condición } \\
\text { y calidad de vida del paciente. El desarrollar la fisiopatología } \\
\text { del craneofaringioma es indispensable, pues permite un ma- } \\
\text { yor entendimiento y la posibilidad de un mejor abordaje para } \\
\text { una patología tan poco común. LUXMÉDICA AÑo 16, NúmER0 47, } \\
\text { MAY0-AGosto 2021, PP 51-59. }\end{array}$ & 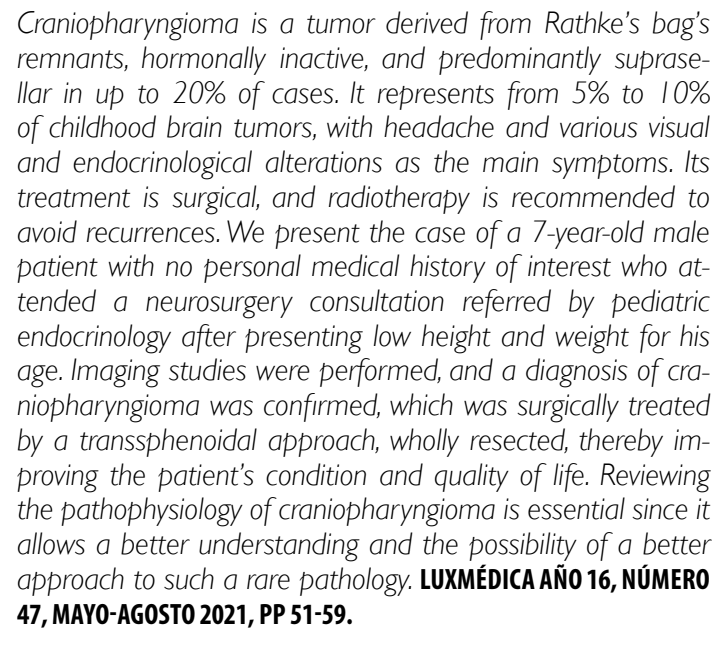 \\
\hline $\begin{array}{r}\text { Palabras clave craneofaringioma, hipertensión intracra- } \\
\text { neal, supraselar }\end{array}$ & $\begin{array}{l}\text { Keywords: craniopharyngioma, intracranial hypertension, } \\
\text { suprasellar }\end{array}$ \\
\hline
\end{tabular}

\section{Introducción}

El craneofaringioma es un tumor de carácter quístico o quístico-sólido, derivado de los remanentes de la bolsa de Rathke. ${ }^{1}$ Son inactivos desde el punto de vista hormonal y se presentan como lesiones cerebrales con predominio supraselar hasta en el $20 \%$ de los casos. ${ }^{2,3}$

\footnotetext{
* Médico Cirujano con especialidad en Neurocirugía. Práctica privada en Hospital Star Médica Aguascalientes. Orcid 0000-0001-7222-1353 Correo electrónico nares.neurospine@gmail.com

** Estudiante del octavo semestre de la Licenciatura de Médico Cirujano del Departamento de Medicina del Centro de Ciencias de la Salud de la Universidad Autónoma de Aguascalientes. Orcid 0000-0002-9668-2354.Correo electrónico rafael.nareslpz@gmail.com

** Estudiante del octavo semestre de la Licenciatura de Médico Cirujano del Departamento de Medicina del Centro de Ciencias de la Salud de la Universidad Autónoma de Aguascalientes. Orcid 0000-0002-8432-9692. Correo electrónico octavioreyesg@hotmail.com

Fecha de recibido: 1 de diciembre 2020

Fecha de aceptación: 5 de marzo 2021

Correspondencia: Dr Felipe Eduardo Nares López. Hospital Star Médica Av. Universidad Nº 101 Fracc. Villas de la Universidad C.P. 20020 Consultorios 211 -212 Aguascalientes, Ags, México. Teléfono 01(449)9129662 Correo electrónico: nares.neurospine@gmail.com
} 
Representa del 5 al 10\% de los tumores cerebrales en la infancia, donde la máxima prevalencia sucede en niños entre 5 y 14 años. ${ }^{1,3}$ La presentación clínica habitual de un craneofaringioma va a depender de múltiples factores, como el volumen de la masa, su ubicación, la edad y probabilidad de crecimiento. Debido a sus características de invasión y expansión puede afectar al sistema hipotálamo-hipofisiario, el sistema ventricular, las vías ópticas y la irrigación sanguínea cerebral. Por lo previamente descrito, se observan signos y síntomas de hipertensión intracraneal, trastornos hormonales por disfunción hipotalámica y alteraciones visuales. Cuando la lesión tiene una localización intraselar, se encuentran similitudes en los signos y síntomas de un adenoma de hipófisis. ${ }^{2}$ Las manifestaciones clínicas varían según la etapa de desarrollo. La hipertensión intracraneal y su manifestación en forma de cefalea, náuseas y vómitos predominan durante la infancia. Las afecciones visuales, si bien son de detección tardía suelen presentarse en forma de hemianopsia o pérdida gradual de la visión. Se ha documentado que en un $90 \%$ de los niños, existe un retraso en el crecimiento como consecuencia de un déficit de hormona de crecimiento y de hormonas tiroideas. A su vez, un $20 \%$ presenta retraso en la pubertad y otro $20 \%$ cursa con diabetes insípida. ${ }^{3}$ De acuerdo con la Organización Mundial de la Salud, existen dos variantes histológicas: adamantinomatosos y papilares, siendo los primeros los más observados en niños y adolescentes. ${ }^{3,4}$

La forma adamantinomatosa presente hasta en el 85\% de los casos, proviene de remanentes epiteliales de tipo ameloblastoma de la cavidad orofaríngea primitiva. Es característico por su organización en cordones o nidos de epitelio escamoso multiestratificado con un tipo de distribución en empalizada. El hallazgo de calcificaciones, acumulación de tipo queratinosa y contenido quístico rico en ácidos grasos y metahemoglobina, se relaciona con esta variante. El tipo papilar, presente en el 15\% restante de los casos, se relaciona con metaplasia de células adenohipofisarias primitivas, con aparición de componentes quísticos en su mayoría. Histológicamente se compone por epitelio escamoso formador de papilas. En esta variante es muy poco frecuente que se encuentren infiltrados o calcificaciones, por tanto la observación de calcificaciones en imágenes son de suma importancia para el diagnóstico diferencial entre las variantes histológicas así como de otras tumoraciones de predominio selar. ${ }^{5}$

Se han observado pronósticos más favorables en la forma papilar con una mejoría en la supervivencia a 5 años en comparación con aquellos que presentan el tipo adamantinomatoso. ${ }^{6,7}$

Para la realización del diagnóstico de un craneofaringioma, es necesario apoyarse con estudios de imagen, siendo el de elección la resonancia magnética (RM). Ésta nos permite conocer específicamente el tamaño y la expansión del tumor a áreas vecinas, así como la identificación de rasgos compatibles con dicha entidad. ${ }^{3}$ Pueden situarse en cualquier lugar del trayecto del conducto craneofaríngeo, encontrándose de manera supraselar hasta en el $75 \%$ de los casos 
seguido de un $21 \%$ de localización selar-supraselar, un $4 \%$ de manera intraselar y sólo el $3.4 \%$ de manera ectópica8. Con base en la literatura revisada, las características que se observan con mayor frecuencia por resonancia magnética en pacientes pediátricos son aquellos tumores multiquísticos de localización supraselar, con una intensidad variable en la secuencia T1. En cambio, en una secuencia T2 tienden a presentarse como hiperintensos posterior al uso de gadolinio. Aunque estas características son poco específicas para determinar el tipo de craneofaringioma a intervenir, se ha demostrado que aquellos craneofaringiomas con presentación lobulada, quística, de gran tamaño, e hiperintensos en T1 son muy sugestivos de ser del tipo adamantinomatoso. Por otra parte, aquellos que son de menor tamaño, forma redondeada, de predominio sólido quísticos, hipointensos en T1, sugieren comúnmente ser del tipo papilar. ${ }^{9}$

El tratamiento de elección es quirúrgico del cual se han propuesto diversos procedimientos, además de recomendarse radioterapia posoperatoria con la intención de evitar recidivas. ${ }^{3}$ La presentación más frecuente de un craneofaringioma es de tipo quística, lo que vuelve complicado una resección total. En diversas series de casos pediátricos, en donde se buscó realizar una resección total, se reportaron tasas de morbilidad y mortalidad de casi $20 \%$, con una tasa de recidiva de entre el $20 \%$ y $50 \%$, además de secuelas endocrinas, neurológicas y oftalmológicas. Es por esto por lo que una gran proporción de los médicos tratantes eligen una combinación de una cirugía menos invasiva, aunado a radioterapia. ${ }^{3}$

Existen factores de buen pronóstico, como la resección total del tumor, la edad de aparición temprana, tamaño menor del tumor, y el tipo histopatológico papilar. Por otra parte, los factores de mal pronóstico incluyen una resección parcial, diseminación e invasión al parénquima vecino que rodea el tumor, y el subtipo histológico adamantinomatoso. ${ }^{10}$ El pronóstico en pacientes que presentan únicamente lesiones de tipo quístico, suele ser mejor que en pacientes con lesiones de características sólidas o mixtas, viéndose reflejado en una sobrevida incrementada. ${ }^{9}$ El objetivo de este trabajo es presentar un caso donde se abordan los problemas más característicos del craneofaringioma, desde una perspectiva fisiopatológica, con el fin de establecer relaciones entre los hallazgos clínicos del paciente y el desarrollo de esta patología poco común.

\section{Presentación de caso clínico}

Se presenta el caso de un paciente masculino de 7 años, sin antecedentes personales patológicos de interés, que acude a la consulta de neurocirugía, referido por endocrinología pediátrica tras estudio de talla y peso bajo para la edad. El paciente inicia su padecimiento actual un año previo con cefalea bifrontal, matutina y progresiva, acompañada de náuseas. Sus padres negaron que el dolor lo despertara por las noches, le produjera vómitos, o se agudizara con cambios de posición. Refieren notar astenia y adinamia. A la exploración física: talla $111 \mathrm{~cm}$, peso $16.400 \mathrm{~kg}$. Resto sin alteraciones. En la exploración neurológica se encontró hemianopsia bitemporal por campimetría. No presenta datos de papiledema. La agudeza visual está conservada. Los movimientos y reflejos oculares normales. Resto sin alteraciones. Se realizan estudios de laboratorio que se muestran en tabla 1. Se comienza tratamiento sustitutivo previo a intervención quirúrgica con levotiroxina $50 \mathrm{mcg}$ al encontrarse tiroxina libre por debajo de rango normal. 


\section{Tabla |}

\section{Resultados de laboratorio}

\begin{tabular}{|l|c|c|}
\multicolumn{1}{c|}{ Hormona } & Resultados & Rango normal \\
\hline Prolactina & $8.47 \mathrm{ng} / \mathrm{ml}$ & $2-18 \mathrm{ng} / \mathrm{ml}$ \\
\hline $\begin{array}{l}\text { Hormona adrenocorticotropa } \\
\text { (ACTH) }\end{array}$ & $14.8 \mathrm{pg} / \mathrm{ml}$ & $7-28 \mathrm{pg} / \mathrm{ml}$ \\
\hline Cortisol & $7.8 \mu \mathrm{g} / \mathrm{dl}$ & $2-18 \mu \mathrm{g} / \mathrm{dl}$ \\
\hline $\begin{array}{l}\text { Hormona del crecimiento (somato- } \\
\text { tropina) }\end{array}$ & $0.787 \mathrm{ng} / \mathrm{ml}$ & $0-20 \mathrm{ng} / \mathrm{ml}$ \\
\hline Tirotropina (TSH) & $3.26 \mu \mathrm{Ul} / \mathrm{ml}$ & $0.8-4.1 \mu \mathrm{Ul} / \mathrm{ml}$ \\
\hline Tiroxina libre (T4L) & 0.57 & $0.8-1.85 \mathrm{ng} / \mathrm{dl}$ \\
\hline
\end{tabular}

\section{Estudios de gabinete}

En el corte sagital de la tomografía de cráneo simple, encontramos una lesión en región selar, con extensión supraselar retroquiasmática, la cual muestra una pseudocápsula tumoral con calcificaciones en periferia y región selar (figura 1). En la resonancia magnética ponderada en T1 corte sagital, línea media, se observa una imagen de $5 \times 5 \times 3 \mathrm{~cm}$ redondeada, homogénea, bien delimitada, en la misma región, que deforma e invade al tercer ventrículo. La lesión es hiperintensa tanto en porción supraselar como selar (figura 2). En la resonancia magnética ponderada en T2 corte sagital, línea media, se observa una imagen de $5 \times 5 \times 3 \mathrm{~cm}$ redondeada, heterogénea, bien delimitada, en región selar con extensión supraselar retroquiasmática que deforma e invade al tercer ventrículo. La lesión es hiperintensa en porción supraselar e isointensa en porción selar (figura 3).

El paciente se sometió a una resección trans-nasal endoscópica con apoyo de neuronavegación. Fue evaluado para tratamiento complementario con el departamento
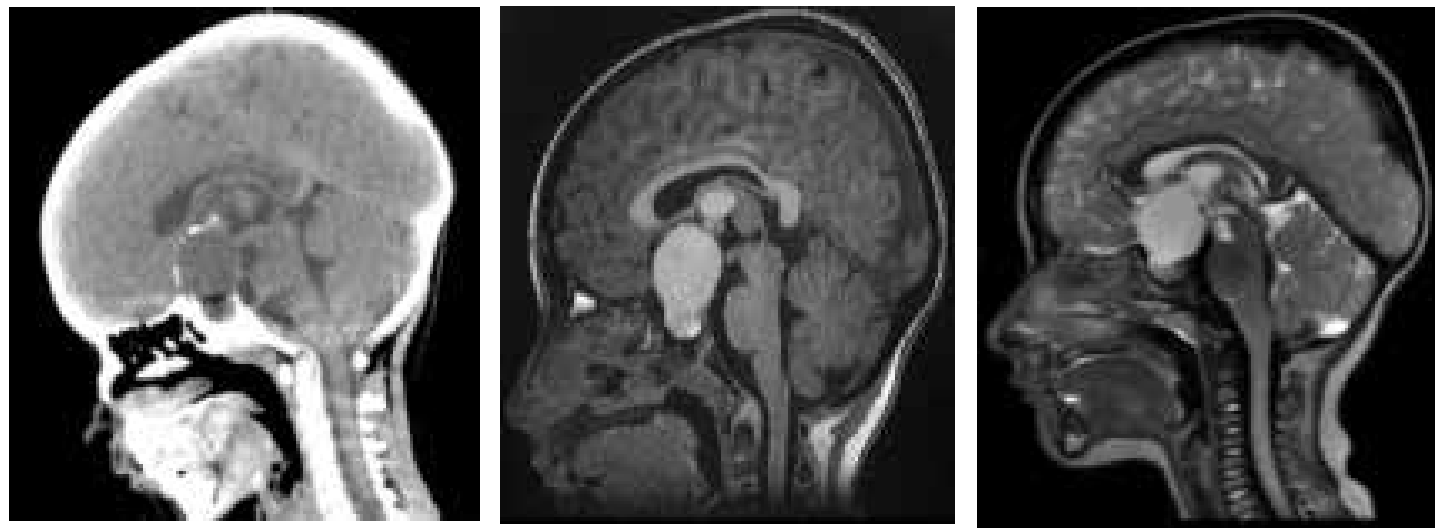

Figura 1.TAC de cráneo simple corte sagital. Figura 2. RM ponderada en T1 corte sagital. Figura 3. RM ponderada en $T 2$ corte sagital (preoperatorias). 
de oncología. El paciente presentó mejoría en los campos visuales por campimetría, de predominio en ojo izquierdo. Los movimientos oculares y agudeza visual se encuentran conservados. El padre refiere mayor interacción social y niega nuevos episodios de cefalea; ha mejorado la astenia y la adinamia. Cursó con diabetes insípida en el postoperatorio inmediato y enuresis, tratado con desmopresina vía oral $1 / 8$ de tableta de 60 mcg. El reporte de histopatología describe abundantes hendiduras de colesterol, células gigantes multinucleadas, queratina y zonas de necrosis, con presencia de calcificaciones, compatible con el diagnóstico de craneofaringioma adamantinomatoso.

En la imagen por resonancia magnética postoperatoria ponderada en T2 (figura 4), se observa la resección de la lesión con preservación del tallo hipofisario, así como cambios quirúrgicos a nivel selar y en seno esfenoidal

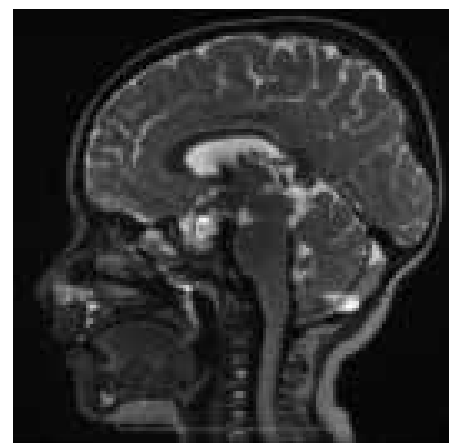

Figura 4. Resonancia magnética ponderada en $T 2$ corte sagital (postoperatoria)

\section{Discusión}

El diagnóstico del craneofaringioma en pacientes pediátricos por lo regular es tardío, pues se ha evidenciado que el promedio de duración de los síntomas antes del diagnóstico es de seis meses, con un margen de 0.1 a 108 meses. $^{5}$
El cuadro clínico que se presenta es consecuencia de la compresión y daño de las estructuras adyacentes que el tumor genera a medida que se expande. Los signos y síntomas más frecuentemente referidos como iniciales son los que se enumeran en la tabla $2 .{ }^{11}$

\section{Tabla 2}

\section{Síntomas y signos más frecuentes de los craneofaringiomas}

\begin{tabular}{|l|c|}
\multicolumn{1}{c|}{ Síntomas y signos } & Frecuencia \\
\hline Neurológicos & \\
Cefalea & $50-75 \%$ \\
Vómitos & $20-35 \%$ \\
Transtornos intelectivos & $20-30 \%$ \\
\hline Déficit motor & $1-3 \%$ \\
\hline Endocrinológicos & \\
Retraso del crecimiento & $10-15 \%$ \\
Diabetes insípida & $5-10 \%$ \\
Obesidad & $3-5 \%$ \\
\hline Pubertad precoz & $1-3 \%$ \\
\hline Visuales & \\
Pérdida de agudeza visual & $15-20 \%$ \\
Estrabismo & $8-13 \%$ \\
\hline
\end{tabular}


Como se ha reportado en la literatura, la primera manifestación que refirió el paciente fue la cefalea de intensidad progresiva, que corresponde a uno de los síntomas de hipertensión intracraneal (HIC). La presión intracraneal se mantiene estable en un rango restringido $(5-15 \mathrm{mmHg})$, a partir de la relación dinámica entre el flujo sanguíneo cerebral, líquido cefalorraquídeo y parénquima cerebral. ${ }^{11}$ La presencia de un volumen agregado en forma de masa conduce a la aparición de HIC precedido de signos y síntomas correspondientes. Generalmente se manifiesta con cefalea, disminución del estado de conciencia y además de déficits neurológicos focales. ${ }^{11}$ La cefalea suele presentarse de forma matutina y en raros casos se localiza en el punto de origen del tumor. ${ }^{12}$ La exploración clínica generalmente revela la existencia de alteraciones en áreas como la visual y la endocrina.

Los trastornos visuales referidos son consecuencia del patrón de crecimiento del craneofaringioma el cual está íntimamente relacionado con el quiasma óptico. La compresión mecánica del quiasma compromete las fibras de los nervios ópticos provenientes de la porción nasal de cada retina, manifestándose como una alteración del campo visual temporal de ambos ojos, originando hemianopsia bitemporal homónima como primer síntoma del síndrome quiasmático. ${ }^{13}$

Al tener una lesión que ocasiona una compresión y desplazamiento de hipófisis e hipotálamo, se espera encontrar un déficit en la secreción y producción de diversas neurohormonas. Tal es el caso de la hormona del crecimiento $(\mathrm{GH})$, que es una de las deficiencias hormonales más comunes cuando se ve afectada la hipófisis anterior $(75 \%){ }^{14}$

La principal función de dicha hormona es favorecer el desarrollo y crecimiento del cuerpo mediante la estimulación del factor de crecimiento tipo insulina I (IGF-I), el cual promueve un incremento en la producción de proteínas al facilitar la obtención de aminoácidos y al actuar directamente en la transcripción y traducción del mRNA. ${ }^{15}$

La diabetes insípida central (DIC) es la consecuencia temprana endócrina más común que se presenta tras la cirugía, se manifiesta en las primeras $24-48$ horas y suele solucionarse durante los siguientes 10 días del postoperatorio. ${ }^{1}$ Cabe mencionar que esta manifestación puede variar con base en el procedimiento realizado por el cirujano pues el $16-66 \%$ de los pacientes que fueron intervenidos por cirugía transcraneal presentan una DIC secundaria, en contraste con un $23-69 \%$ de los pacientes que son intervenidos por cirugía transesfenoidal. 1,16

La DIC es un trastorno distinguido por la deficiencia de hormona antidiurética (ADH) que ocasiona poliuria y polidipsia. Puede presentarse de manera primaria o como en este caso, secundaria al trauma derivado de la intervención quirúrgica lo que ocasiona degeneración neuronal de los núcleos supraóptico y paraventricular hipotalámicos, encargados de regular la secreción de ADH dada por la neurohipófisis. ${ }^{16}$ De no reponer los líquidos de manera adecuada, puede conducir a sintomatología neurológica. El diagnóstico considera $>100 \mathrm{ml} / \mathrm{kg} /$ día, osmolalidad urinaria $<100 \mathrm{mOsm} / \mathrm{kg}$ o inferior a la plasmática, osmolalidad sérica aumentada. ${ }^{17}$ Dependiendo de la gravedad del cuadro clínico está indicado en pacientes pediátricos el tratamiento con desmopresina vía oral $0.1 \mathrm{mg}$, comúnmente en dosis única. ${ }^{18}$

Cuando se realiza la resección de un craneofaringioma, se vuelve de alta relevancia el abordaje del tumor que, en el presente caso, fue transesfenoidal con neuronavegación. La literatura actual señala dicho abordaje como el más seguro para el paciente en cuestión, de preservación hipotalámica y por lo tanto calidad de vida; ${ }^{19}$ la visión y el estado endocrinológico son las mayores preocupaciones referentes a la calidad de vida de los pacientes posterior a la cirugía (tabla 3). Múltiples factores pueden contribuir al estado de la visión, como lo son las 
afectaciones previas a la cirugía, una adhesión de amplia extensión entre el tumor y el nervio óptico, quiasma y tracto óptico, además de calcificación del tumor. ${ }^{3}$

Se ha encontrado que el deterioro visual postoperatorio se presenta de manera más frecuente en el abordaje pterional, por lo que se recomienda un abordaje con métodos de mínima invasión. ${ }^{20}$

El craneofaringioma está relacionado con excesiva morbilidad multisistémica a largo plazo, en gran medida relacionado al hipopituitarismo. Estos tumores usualmente afectan completamente la secreción adenohipofisaria por compresión directa o destrucción del hipotálamo y el tallo hipofisario. Sin embargo, las manifestaciones clínicas pueden no ser muy obvias. En una serie de 309 casos realizada por Shi y cols. ${ }^{20}$ la mayoría de los niveles hormonales se encontraban dentro de rangos normales previo a la cirugía. Dado el riesgo que se tiene de dañar directamente el eje hipotálamo-hipófisis durante el abordaje es importante mantener las concentraciones normales de las distintas hormonas, previniendo cualquier alteración endocrina. Por esto se debe de tener especial atención en la preservación de estas estructuras, tomando en consideración que las cirugías por recidiva suelen tener una mortalidad mayor; cabe mencionar que es sumamente raro observar una reversión del daño posterior a la cirugía. ${ }^{21}$ Dicha situación, vuelve compleja la toma de decisión respecto al abordaje y agresividad en la cirugía. A pesar de una resección satisfactoria, se encuentran déficits hormonales más evidentes que previo a la cirugía, incluso con el $60.2 \%$ del tallo hipofisario preservado. ${ }^{20}$ Por lo tanto, un completo asesoramiento endocrinológico es necesario, en especial por alteraciones en $\mathrm{GH}$, que es la deficiencia endocrina más común en niños y adolescentes.

\section{Tabla 3}

\section{Prevalencia de complicaciones postoperatorias a un mes}

\begin{tabular}{|l|c|c|}
\hline \multicolumn{1}{|c|}{ Complicaciones } & Casos totales $(\mathbf{N}=309)$ & Casos recuperados \\
\hline Diabetes insípida & $163(52.8 \%)$ & $79(48.5 \%)$ \\
\hline SIADH & $90(29.1 \%)$ & $90(100 \%)$ \\
\hline Hipernatremia & $77(25.0 \%)$ & $69(89.1 \%)$ \\
\hline Deterioro visual & $17(5.5 \%)$ & $11(64.7 \%)$ \\
\hline Hiperpirexia y coma & $8(2.6 \%)$ & $3(37.5 \%)$ \\
\hline Parálisis de extremidad & $4(1.3 \%)$ & $2(50 \%)$ \\
\hline SPSC & $3(1.0 \%)$ & $2(66.7 \%)$ \\
\hline Parálisis N. oculomotor & $3(1.0 \%)$ & 0 \\
\hline Hematoma intraventricular & $1(0.3 \%)$ & 0 \\
\hline Hematoma supraselar & $1(0.3 \%)$ & \\
\hline Muerte en perioperatorio & $12(3.8 \%)$ & $2 \%)$ \\
\hline
\end{tabular}

Nota: estas complicaciones pueden sobreponerse en un mismo paciente. SIADH: Síndrome de secreción inadecuada de hormona antidiurética. SPSC: Síndrome pierde sal cerebral 
Otro punto clave en el abordaje es, la decisión de dar radioterapia como complemento con la intención de evitar recidivas y las complicaciones previamente mencionadas. La utilización de radioterapia en niños siempre ha representado un dilema terapéutico, debido a los riesgos de toxicidad a largo plazo, por lo que se necesita realizar un balance entre los riesgos de recidiva y los riesgos de dicha toxicidad. Si bien está reportado el daño que la radiación provoca en pacientes con craneofaringioma a nivel endocrino o cognitivo, éstos suelen ser menores que en pacientes que reciben una resección completa del tumor de manera agresiva, comúnmente por un abordaje subfrontal22. Por otra parte, el tiempo de aplicación de la radioterapia continúa sien- do tema de discusión. Regine y Kramer ${ }^{23}$ realizaron un estudio que mostró que los pacientes que fueron tratados con radioterapia de manera inicial tuvieron un índice de supervivencia a 5 años de $78 \%$ comparado con $25 \%$ en pacientes que recibieron radioterapia únicamente al momento de recurrencia. Por su parte Lin y cols. ${ }^{22}$ realizaron un seguimiento a largo plazo donde se mostró que el uso de radioterapia en pacientes en quienes se realizó una resección parcial del tumor mejora el pronóstico a pesar de no tener una resección completa, sobretodo en cuestión de recidiva a 5 años, razón por la cual se recomienda actualmente radioterapia como tratamiento paralelo a la cirugía. ${ }^{24}$

\section{Conclusiones}

El craneofaringioma es un tumor de origen epitelial de la región selar que se forma a partir de los restos embrionarios de la bolsa de Rathke, según su patrón de crecimiento puede limitarse a la silla turca o bien puede extenderse a través del diafragma selar y comprimir estructuras adyacentes, dando como resultado múltiples síntomas que abarcan alteraciones visuales, endocrinas y neurológicas. Conocer el desarrollo y manejo de esta entidad permite al médico llevar a cabo una detección oportuna para optar por el abordaje más eficaz, y beneficioso para el paciente, previniendo al máximo las complicaciones que puedan surgir y las secuelas que dicha alteración pueda generar, de manera que se mejore la calidad de vida del paciente.

\section{Bibliografía}

1. Tena-Suck Martha Lilia, Moreno-Reyes Ignacio, RembaoBojórquez Daniel, Vega Rosalba, Moreno-Jiménez Sergio, Castillejos-López Manuel de Jesús, Fernández-Plata María del Rosario, Martínez-Briseño David, Salinas-Lara Citlaltepetl. Craneofaringioma, estudio clínico-patológico. Quince años del Instituto Nacional de Neurología y Neurocirugía "Manuel Velasco Suárez". Gaceta Médica de México. 2009;145(5):361-368.

2. Díaz-Pérez JA, Duran-Rodríguez-Hervada, Runkle de la Vega I, de Miguel Novoa M. Panhipopituitarismo. Medicine - Programa de Formación Médica Continuada Acreditado. 2004;9(13):782-790. DOI: https://doi.org/10.1016/ S0211-3449(04)70111-3.

3. Páramo-Fernández Concepción, Picó-Alfonso Antonio Miguel, del Pozo-Picó Carlos, Varela-da Costa César, Lucas-
Morante Tomás, Catalá-Bauset Miguel, Gilsanz-Peral Alberto, Halperin-Rabinovich Irene, Moreno-Esteban Basilio, Obiols-Alfonso Gabriel, Torres-Vela Elena, Tortosa-Henzi Frederic, Webb S. M, Zugasti-Murillo Ana, VillabonaArtero Carles. Guía clínica del diagnóstico y tratamiento del craneofaringioma y otras lesiones paraselares. Endocrinología y Nutrición. 2007;54(1):13-22. DOI: https://doi. org/10.1016/S1575-0922(07)71401-8

4. Lamas-Oliveira Cristina. Consecuencias metabólicas del craneofaringioma y su tratamiento. Endocrinología y Nutrición. 2013;60(9):529-534.

DOI: 10.1016/j.endonu.2012.11.008.

5. Cabrera-Bernal E. Craneofaringioma en pediatría: Diagnóstico y tratamiento. Rev.Col.Hematol.Oncol.2020;7(1).

6. Baraquiso Pazos et al. Actualización de craneofaringioma, revisión de tema.Update in craniopharyngioma, a review of literature. Ciencia y salud. 2020;4(6):7-13. https://doi. 
org/10.34192/cienciaysalud.v4i6.23

7. Müller-Hermann L, Merchant-Thomas E, Warmuth-Metz Monika, Martínez-Barbera Juan-Pedro, Puget Stephanie. Craniopharyngioma. Nature Reviews-Desease Primers. 2019; 5(75):1-19 https://doi.org/10.1038/s41572-0190125-9

8. Sánchez I, Pérez R, Ortiz M, Aguilar M, Quevedo N. Correlación de evaluaciones clínicas e imagenológicos en casos con craneofaringioma. Revista Clínica de la Escuela de Medicina UCR-HSJD.2020; 10(3): 48-54.

9. Robles V, Horta A, Franco R. Características por resonancia magnética del craneofaringioma. Experiencia en ocho pacientes pediátricos en el Hospital General del Centro Médico Nacional "La Raza". Anales de Radiología México. 2010;4:239-245.

10. Martínez-González M, García-Ribes A, Garaizar-Axpe C. Tumores cerebrales infantiles: Diagnóstico y semiología neurológica. Protocolo Diagnóstico Terapéuticos de la AEP: Neurología Pediátrica. 2008;27:203-209.

11. Gilo-Arrojo F, Herrera-Muñoz A, Anciones B. Hipertensión intracraneal aguda. Neurología. 2010;25:3-10. DOI: https://doi.org/10.1016/S0213-4853(10)70044-X

12. Arjona-Villanueva $D$, Borrego-Domínguez $R$, Huidobro $B$, Fernández-Barrio B, Verdú-Pérez A. Hipertensión Intracraneal. Protocolos Diagnóstico-Terapéuticos de la AEP: Neurología Pediátrica. 2008;33:244-254.

13. Padilla $H$, Ramos $Y$, Pereira J, Valencia L, Manjarrez J, Corrales $\mathrm{H}$, et al. Síndrome Quiasmático. Revisión bibliográfica. Revista Latinoamericana de Neurocirugía. 2017;26(3).

14. Peralta Watt $M$, Peñalver Talavera D. Panhipopituitarismo por craneofaringioma asociado a hipertiroidismo por enfermedad de Graves-Basedow. Endocrinología y Nutrición. 2008;55(7):297-300. DOI: https://doi.org/10.1016/S1575-0922(08)72184-3

15. Gardner DG, Shoback D, Muñoz BR. Hipotálamo y Glándula Hipófisis. Greenspan. Endocrinología básica y clínica. McGraw-Hill Interamericana Editores. 2012:65-90

16. Loureiro PC, Martínez AA, Fernández BR, Lizama $C M$, Cattani OA, García BH. Diabetes Insípida en Pediatría: Serie Clínica y Revisión de la Literatura. Rev. Chil. Pediatr. 2009;80(3):245-255. DOI: http://dx.doi.org/10.4067/ S0370-41062009000300006
17. Lamas C, del Pozo C, Villabona C. Guía clínica de manejo de la diabetes insípida y del síndrome de secreción inapropiada de hormona antidiurética en el postoperatorio de la cirugía hipofisaria. Endocrinología y Nutrición. 2014;61(4):15-24. DOI:https://doi.org/10.1016/j.endonu.2014.01.005

18. Hannon MJ, Finucane FM, Sherlock M, Agha A, Thompson CJ. Disorders of water Homeostasis in Neurosurgical Patients. The journal of Clinical Endocrinology \& Metabolism. 2012;97(5):1423-33. DOI: https://doi.org/10.1210/ jc.2011-3201

19. Yano S, Hide T, Shinojima N. Surgical Outcomes of Endoscopic Endonasal Skull Base Surgery of Craniopharyngiomas Evaluated According to the Degree of Hypothalamic Extension. World Neurosurg. 2017 Apr;100:288-296. https://doi.org/10.1016/j.wneu.2017.01.005

20. Shi XE, Wu B, Fan T, Zhou ZQ, Zhang YL. Craniopharyngioma: surgical experience of 309 cases in China. Clin Neurol Neurosurg 2008;110:151-9.DOI: https://doi. org/10.1016/j.clineuro.2007.10.013

21. Fomichev $D$, Kalinin $P$, Kutin $M$, Sharipov O. Extended Transsphenoidal Endoscopic Endonasal Surgery of Suprasellar Craniopharyngiomas. World Neurosurg. 2016 Oct;94:181-187. https://doi.org/10.1016/j. wneu.2016.06.124

22. Lin LL, Naqa I, Leonard JR, Park T, Hollander AS, Michalski $J M$, Mansur DB. Long-term outcome in children treated for craniopharyngioma with and without radiotherapy, Journal of Neurosurgery: Pediatrics PED, 2008;1(2):126-130. DOI: https://doi.org/10.3171/PED/2008/1/2/126

23. Regine WF, Kramer S. Pediatric craniopharyngiomas: long term results of combined treatment with surgery and radiation. Int J Radiat Oncol Biol Phys. 1992;24(4):611-7. DOI: https://doi.org/10.1016/0360-3016(92)90705-M

24. Venegas $E$, Concepcion B, Martin T, Soto A. Guía práctica del manejo y tratamiento de los craneofaringiomas y otras lesiones paraselares [Practice guideline for diagnosis and treatment of craniopharyngioma and parasellar tumors of the pituitary gland]. Endocrinol Nutr. 2015 Jan;62(1):e1-13. Spanish. https://doi.org/10.1016/j.endonu.2014.05.005 